\title{
Zs Research foure \\ The Association Between Household Income, Food Security, and Prevalence of Chronic Kidney Disease in Elderly \\ Patients
}

Hyuk Huh

Seoul National University Hospital

Je Hun Song

University of Ulsan College of Medicine

Hong Yeop Kim

University of Ulsan College of Medicine

Hoseok Koo

Inje University College of Medicine

Kyung Don Yoo ( $\nabla$ ykd9062@gmail.com )

University of Ulsan College of Medicine

\section{Research Article}

Keywords: prevalence of hypertension, diabetes mellitus (DM), metabolic syndrome, and chronic kidney disease (CKD), ESRD

Posted Date: September 20th, 2021

DOI: https://doi.org/10.21203/rs.3.rs-913699/v1

License: (c) (i) This work is licensed under a Creative Commons Attribution 4.0 International License. Read Full License 


\section{Abstract}

This study aimed to clarify the association between food security and the prevalence of chronic disease. We analyzed the variables of The Korea National Health and Nutrition Examination Survey V (2010-2012), and VI (2013-2015) while merging data of the food security questionnaire of four years. We included 15,945 participants, performed propensity score matched analysis by quartile of household income (i.e., low, low-mid, high-mid, high) and sex, and presented the results by age group. Systolic blood pressure and proportion of current smokers were significantly higher in the elderly group, compared with the middle-aged group. The prevalence of hypertension, diabetes mellitus (DM), metabolic syndrome, and chronic kidney disease (CKD) did not differ significantly by income level in the elderly group. The food security questionnaire revealed that food security insurance was significantly lower in the low-income level (1st quartile), compared with that in the high-income level (4th quartile). The logistic regression analysis for the association between the prevalence of chronic disease and food insecurity confirmed no significant association with hypertension and DM. Food insecurity might be associated with CKD prevalence, especially in the elderly population.

\section{Introduction}

Several studies have shown a varying prevalence of chronic diseases and inequality in life expectancy by socioeconomic status in western societies. Many researchers have confirmed a higher prevalence and mortality of several metabolic diseases in socioeconomically vulnerable groups [1, 2]. Stratification of socioeconomic status reflects the differentiation in factors such as health behaviors, food security, health risk behaviors, and educational, occupational, and income levels. Among them, food security is defined as the ability to consume a balanced diet of nutrients, fruits, and vegetables, while limiting salt, saturated fat, and carbohydrates. In recent times, there have been studies on the relationship between food insecurity and chronic diseases such as hypertension, diabetes, cardiovascular disease, and chronic kidney disease (CKD). Deidra [3] showed an association between food insecurity and CKD in diabetic and hypertensive patients in the United States, whereas Banerjee [4] revealed that food instability is an independent risk factor for the development of endstage renal disease (ESRD) in patients with CKD. The latter study was based on data from the American Health and Nutrition Examination Survey. Although we cannot conclude that food insecurity has a powerful effect like other known risk factors for chronic diseases such as smoking or alcohol consumption, it may affect vulnerable groups including patients with chronic disease and the elderly. In line with this, a study on the Japanese population suggests an association between lower income levels and higher risks of CKD [5].

However, few studies focus on the contribution of education and income level, and food security to the prevalence of chronic diseases in the Korean population. Therefore, this study confirmed the effect of income level and food safety on the health status of Koreans based on data from the Korean National Health and Nutrition Examination Survey (KNHANES).

\section{Results}

\section{Baseline characteristics.}

Participants' basic characteristics are shown in Table 1. The middle-aged and elderly groups were matched by the propensity score of 3,957 people. According to their income level, they were divided into quartiles comprising 921 people in the low group, 999 people in the low-mid group, 1012 people in the high-mid group, and 1025 people in the high group. 
Table 1

Baseline characteristics by household income level after propensity score matching.

\begin{tabular}{|c|c|c|c|c|c|c|c|c|c|c|}
\hline & \multicolumn{5}{|c|}{ Middle-aged group } & \multicolumn{5}{|c|}{ Elderly group } \\
\hline & Low & $\begin{array}{l}\text { Low- } \\
\text { mid }\end{array}$ & $\begin{array}{l}\text { High- } \\
\text { mid }\end{array}$ & High & $\begin{array}{l}\mathrm{P}- \\
\text { value }\end{array}$ & Low & $\begin{array}{l}\text { Low- } \\
\text { mid }\end{array}$ & $\begin{array}{l}\text { High- } \\
\text { mid }\end{array}$ & High & $\begin{array}{l}\mathrm{P} \text { - } \\
\text { value }\end{array}$ \\
\hline & $\begin{array}{l}(\mathrm{N}= \\
921)\end{array}$ & $\begin{array}{l}(\mathrm{N}= \\
999)\end{array}$ & $\begin{array}{l}(\mathrm{N}= \\
1012)\end{array}$ & $\begin{array}{l}(\mathrm{N}= \\
1025)\end{array}$ & & $\begin{array}{l}(\mathrm{N}= \\
921)\end{array}$ & $\begin{array}{l}(\mathrm{N}= \\
999)\end{array}$ & $\begin{array}{l}(\mathrm{N}= \\
1012)\end{array}$ & $\begin{array}{l}(\mathrm{N}= \\
1025)\end{array}$ & \\
\hline Age & $\begin{array}{l}44.0 \pm \\
12.6\end{array}$ & $\begin{array}{l}44.3 \pm \\
12.5\end{array}$ & $\begin{array}{l}43.9 \pm \\
12.4\end{array}$ & $\begin{array}{l}44.8 \pm \\
12.6\end{array}$ & 0.194 & $\begin{array}{l}71.9 \pm \\
4.6\end{array}$ & $\begin{array}{l}72.0 \pm \\
4.8\end{array}$ & $\begin{array}{l}71.7 \pm \\
4.7\end{array}$ & $\begin{array}{l}71.6 \pm \\
4.7\end{array}$ & 0.047 \\
\hline Sex & & & & & 0.817 & & & & & 0.817 \\
\hline Male & $\begin{array}{l}415 \\
(45.1 \%)\end{array}$ & $\begin{array}{l}436 \\
(43.6 \%)\end{array}$ & $\begin{array}{l}460 \\
(45.5 \%)\end{array}$ & $\begin{array}{l}450 \\
(43.9 \%)\end{array}$ & & $\begin{array}{l}415 \\
(45.1 \%)\end{array}$ & $\begin{array}{l}436 \\
(43.6 \%)\end{array}$ & $\begin{array}{l}460 \\
(45.5 \%)\end{array}$ & $\begin{array}{l}450 \\
(43.9 \%)\end{array}$ & \\
\hline Female & $\begin{array}{l}506 \\
(54.9 \%)\end{array}$ & $\begin{array}{l}563 \\
(56.4 \%)\end{array}$ & $\begin{array}{l}552 \\
(54.5 \%)\end{array}$ & $\begin{array}{l}575 \\
(56.1 \%)\end{array}$ & & $\begin{array}{l}506 \\
(54.9 \%)\end{array}$ & $\begin{array}{l}563 \\
(56.4 \%)\end{array}$ & $\begin{array}{l}552 \\
(54.5 \%)\end{array}$ & $\begin{array}{l}575 \\
(56.1 \%)\end{array}$ & \\
\hline SBP & $\begin{array}{l}116.4 \\
\pm 15.6\end{array}$ & $\begin{array}{l}116.3 \\
\pm 15.7\end{array}$ & $\begin{array}{l}115.8 \\
\pm 15.4\end{array}$ & $\begin{array}{l}115.1 \\
\pm 15.8\end{array}$ & 0.063 & $\begin{array}{l}128.9 \\
\pm 17.0\end{array}$ & $\begin{array}{l}129.2 \\
\pm 18.1\end{array}$ & $\begin{array}{l}127.7 \\
\pm 16.5\end{array}$ & $\begin{array}{l}128.4 \\
\pm 17.3\end{array}$ & 0.236 \\
\hline DBP & $\begin{array}{l}75.9 \pm \\
10.4\end{array}$ & $\begin{array}{l}75.9 \pm \\
10.3\end{array}$ & $\begin{array}{l}76.1 \pm \\
10.5\end{array}$ & $\begin{array}{l}75.5 \pm \\
10.4\end{array}$ & 0.535 & $\begin{array}{l}73.5 \pm \\
10.1\end{array}$ & $\begin{array}{l}72.8 \pm \\
10.4\end{array}$ & $\begin{array}{l}72.8 \pm \\
9.7\end{array}$ & $\begin{array}{l}73.2 \pm \\
9.5\end{array}$ & 0.635 \\
\hline WC & $\begin{array}{l}81.0 \pm \\
10.0\end{array}$ & $\begin{array}{l}80.8 \pm \\
9.6\end{array}$ & $\begin{array}{l}80.7 \pm \\
9.6\end{array}$ & $\begin{array}{l}79.8 \pm \\
9.6\end{array}$ & 0.005 & $\begin{array}{l}84.5 \pm \\
9.2\end{array}$ & $\begin{array}{l}83.9 \pm \\
9.0\end{array}$ & $\begin{array}{l}84.6 \pm \\
8.9\end{array}$ & $\begin{array}{l}84.3 \pm \\
8.9\end{array}$ & 0.880 \\
\hline BMI & $\begin{array}{l}23.9 \pm \\
3.7\end{array}$ & $\begin{array}{l}23.9 \pm \\
3.4\end{array}$ & $\begin{array}{l}23.8 \pm \\
3.3\end{array}$ & $\begin{array}{l}23.5 \pm \\
3.3\end{array}$ & 0.01 & $\begin{array}{l}24.0 \pm \\
3.2\end{array}$ & $\begin{array}{l}23.9 \pm \\
3.1\end{array}$ & $\begin{array}{l}24.1 \pm \\
3.0\end{array}$ & $\begin{array}{l}24.1 \pm \\
3.1\end{array}$ & 0.127 \\
\hline $\begin{array}{l}\text { Total } \\
\text { cholesterol }\end{array}$ & $\begin{array}{l}188.6 \\
\pm 35.5\end{array}$ & $\begin{array}{l}191.1 \\
\pm 36.8\end{array}$ & $\begin{array}{l}189.7 \\
\pm 34.7\end{array}$ & $\begin{array}{l}190.6 \\
\pm 35.2\end{array}$ & 0.38 & $\begin{array}{l}189.0 \\
\pm 38.9\end{array}$ & $\begin{array}{l}187.9 \\
\pm 36.4\end{array}$ & $\begin{array}{l}188.3 \\
\pm 38.1\end{array}$ & $\begin{array}{l}185.8 \\
\pm 35.4\end{array}$ & 0.084 \\
\hline $\begin{array}{l}\text { MDRD } \\
\text { eGFR }\end{array}$ & $\begin{array}{l}97.0 \pm \\
18.8\end{array}$ & $\begin{array}{l}96.6 \pm \\
17.4\end{array}$ & $\begin{array}{l}96.3 \pm \\
18.4\end{array}$ & $\begin{array}{l}94.8 \pm \\
17.2\end{array}$ & 0.008 & $\begin{array}{l}83.6 \pm \\
17.5\end{array}$ & $\begin{array}{l}82.9 \pm \\
18.0\end{array}$ & $\begin{array}{l}83.3 \pm \\
18.1\end{array}$ & $\begin{array}{l}81.7 \pm \\
18.1\end{array}$ & 0.034 \\
\hline $\begin{array}{l}\text { Current } \\
\text { smoker }\end{array}$ & $\begin{array}{l}244 \\
(26.5 \%)\end{array}$ & $\begin{array}{l}227 \\
(22.7 \%)\end{array}$ & $\begin{array}{l}222 \\
(21.9 \%)\end{array}$ & $\begin{array}{l}175 \\
(17.1 \%)\end{array}$ & $<.001$ & $\begin{array}{l}123 \\
(13.4 \%)\end{array}$ & $\begin{array}{l}101 \\
(10.1 \%)\end{array}$ & $\begin{array}{l}111 \\
(11.0 \%)\end{array}$ & $\begin{array}{l}73 \\
(7.1 \%)\end{array}$ & 0.000 \\
\hline Education & & & & & 0.000 & & & & & 0.000 \\
\hline $\begin{array}{l}\text { Elementary } \\
\text { school }\end{array}$ & $\begin{array}{l}183 \\
(19.9 \%)\end{array}$ & $\begin{array}{l}143 \\
(14.3 \%)\end{array}$ & $\begin{array}{l}88 \\
(8.7 \%)\end{array}$ & $\begin{array}{l}55 \\
(5.4 \%)\end{array}$ & & $\begin{array}{l}680 \\
(73.8 \%)\end{array}$ & $\begin{array}{l}698 \\
(69.9 \%)\end{array}$ & $\begin{array}{l}585 \\
(57.8 \%)\end{array}$ & $\begin{array}{l}443 \\
(43.2 \%)\end{array}$ & \\
\hline $\begin{array}{l}\text { Middle } \\
\text { school }\end{array}$ & $\begin{array}{l}120 \\
(13.0 \%)\end{array}$ & $\begin{array}{l}101 \\
(10.1 \%)\end{array}$ & $\begin{array}{l}85 \\
(8.4 \%)\end{array}$ & $\begin{array}{l}58 \\
(5.7 \%)\end{array}$ & & $\begin{array}{l}107 \\
(11.6 \%)\end{array}$ & $\begin{array}{l}138 \\
(13.8 \%)\end{array}$ & $\begin{array}{l}165 \\
(16.3 \%)\end{array}$ & $\begin{array}{l}131 \\
(12.8 \%)\end{array}$ & \\
\hline $\begin{array}{l}\text { High } \\
\text { school }\end{array}$ & $\begin{array}{l}393 \\
(42.7 \%)\end{array}$ & $\begin{array}{l}411 \\
(41.1 \%)\end{array}$ & $\begin{array}{l}395 \\
(39.0 \%)\end{array}$ & $\begin{array}{l}357 \\
(34.8 \%)\end{array}$ & & $\begin{array}{l}102 \\
(11.1 \%)\end{array}$ & $\begin{array}{l}127 \\
(12.7 \%)\end{array}$ & $\begin{array}{l}188 \\
(18.6 \%)\end{array}$ & $\begin{array}{l}254 \\
(24.8 \%)\end{array}$ & \\
\hline College & $\begin{array}{l}225 \\
(24.4 \%)\end{array}$ & $\begin{array}{l}344 \\
(34.4 \%)\end{array}$ & $\begin{array}{l}444 \\
(43.9 \%)\end{array}$ & $\begin{array}{l}555 \\
(54.1 \%)\end{array}$ & & $\begin{array}{l}32 \\
(3.5 \%)\end{array}$ & $\begin{array}{l}36 \\
(3.6 \%)\end{array}$ & $\begin{array}{l}74 \\
(7.3 \%)\end{array}$ & $\begin{array}{l}197 \\
(19.2 \%)\end{array}$ & \\
\hline
\end{tabular}

In the middle age group, no differences in systolic and diastolic blood pressure according to income level were found. Abdominal circumference and BMI tended to be higher among those with lower income levels. Further, the Modification of Diet in Renal Disease (MDRD) eGFR and proportion of smokers was higher among those with lower income levels. Regarding educational level, higher income levels were significantly positively associated with university education, and negatively associated with elementary school education. 
In the elderly age group, no significant differences in blood pressure, abdominal circumference, BMI, and total cholesterol

level were found by income level. Lower income levels were associated with higher MDRD eGFR. Further, educational level and smoking rate showed similar trends by income level, as in the middle-aged group.

\section{Prevalence of chronic disease and medical service needs.}

In the middle age group, lower income levels were associated with a higher prevalence of hypertension, diabetes, and metabolic syndrome. The prevalence of hypertension was $17.5 \%$ and $24.0 \%$ in high- and low-income groups respectively. Additionally, $6.3 \%$ and $10.0 \%$ of patients in the high- and low-income group were diagnosed with diabetes, respectively. However, the prevalence of CKD did not differ between groups. Regarding the lack of access to necessary medical services due to economic reasons, $0.5 \%$ and $7.6 \%$ of those in the high- and low-income groups, respectively did not receive essential medical services owing to economic reasons (Table 2). However, no statistically significant difference was observed for this variable. 
Table 2

Prevalence of chronic disease and medical service needs by household incomes between the age groups.

\begin{tabular}{|c|c|c|c|c|c|}
\hline & Low & Low-mid & High-mid & High & $\begin{array}{l}\mathrm{P} \text { - } \\
\text { value }\end{array}$ \\
\hline Middle-age group ( $\mathrm{N}=3957$ ) & $\begin{array}{l}(\mathrm{N}= \\
921)\end{array}$ & $(\mathrm{N}=999)$ & $\begin{array}{l}(\mathrm{N}= \\
1012)\end{array}$ & $\begin{array}{l}(\mathrm{N}= \\
1025)\end{array}$ & \\
\hline \multicolumn{6}{|l|}{ Chronic disease } \\
\hline Hypertension & $\begin{array}{l}221 \\
(24.0 \%)\end{array}$ & $\begin{array}{l}209 \\
(20.9 \%)\end{array}$ & $\begin{array}{l}214 \\
(21.1 \%)\end{array}$ & $\begin{array}{l}179 \\
(17.5 \%)\end{array}$ & 0.012 \\
\hline DM & $\begin{array}{l}92 \\
(10.0 \%)\end{array}$ & $\begin{array}{l}79 \\
(7.9 \%)\end{array}$ & $\begin{array}{l}66 \\
(6.5 \%)\end{array}$ & $65(6.3 \%)$ & 0.013 \\
\hline CKD & $\begin{array}{l}12 \\
(1.3 \%)\end{array}$ & $\begin{array}{l}11 \\
(1.1 \%)\end{array}$ & $8(0.8 \%)$ & $12(1.2 \%)$ & 0.161 \\
\hline Metabolic syndrome & $\begin{array}{l}133 \\
(14.4 \%)\end{array}$ & $\begin{array}{l}119 \\
(11.9 \%)\end{array}$ & $\begin{array}{l}128 \\
(12.6 \%)\end{array}$ & $98(9.6 \%)$ & 0.010 \\
\hline Metabolic syndrome score & & & & & 0.002 \\
\hline 0 & $\begin{array}{l}368 \\
(40.0 \%)\end{array}$ & $\begin{array}{l}415 \\
(41.5 \%)\end{array}$ & $\begin{array}{l}421 \\
(41.6 \%)\end{array}$ & $\begin{array}{l}496 \\
(48.4 \%)\end{array}$ & \\
\hline 1 & $\begin{array}{l}255 \\
(27.7 \%)\end{array}$ & $\begin{array}{l}287 \\
(28.7 \%)\end{array}$ & $\begin{array}{l}271 \\
(26.8 \%)\end{array}$ & $\begin{array}{l}270 \\
(26.3 \%)\end{array}$ & \\
\hline 2 & $\begin{array}{l}165 \\
(17.9 \%)\end{array}$ & $\begin{array}{l}178 \\
(17.8 \%)\end{array}$ & $\begin{array}{l}192 \\
(19.0 \%)\end{array}$ & $\begin{array}{l}161 \\
(15.7 \%)\end{array}$ & \\
\hline 3 & $\begin{array}{l}109 \\
(11.8 \%)\end{array}$ & $\begin{array}{l}95 \\
(9.5 \%)\end{array}$ & $\begin{array}{l}90 \\
(8.9 \%)\end{array}$ & $74(7.2 \%)$ & \\
\hline 4 & $\begin{array}{l}24 \\
(2.6 \%)\end{array}$ & $\begin{array}{l}24 \\
(2.4 \%)\end{array}$ & $\begin{array}{l}38 \\
(3.8 \%)\end{array}$ & $24(2.3 \%)$ & \\
\hline $\begin{array}{l}\text { Lack of access to necessary medical services owing to } \\
\text { economic reasons }\end{array}$ & $\begin{array}{l}70 \\
(7.6 \%)\end{array}$ & $\begin{array}{l}25 \\
(2.5 \%)\end{array}$ & $\begin{array}{l}20 \\
(2.0 \%)\end{array}$ & $5(0.5 \%)$ & 0.730 \\
\hline Elderly group ( $\mathrm{N}=3957)$ & $\begin{array}{l}(\mathrm{N}= \\
921)\end{array}$ & $(\mathrm{N}=999)$ & $\begin{array}{l}(\mathrm{N}= \\
1012)\end{array}$ & $\begin{array}{l}(\mathrm{N}= \\
1025)\end{array}$ & \\
\hline \multicolumn{6}{|l|}{ Chronic disease } \\
\hline Hypertension & $\begin{array}{l}555 \\
(60.3 \%)\end{array}$ & $\begin{array}{l}629 \\
(63.0 \%)\end{array}$ & $\begin{array}{l}616 \\
(60.9 \%)\end{array}$ & $\begin{array}{l}621 \\
(60.6 \%)\end{array}$ & 0.611 \\
\hline DM & $\begin{array}{l}222 \\
(24.1 \%)\end{array}$ & $\begin{array}{l}210 \\
(21.0 \%)\end{array}$ & $\begin{array}{l}233 \\
(23.0 \%)\end{array}$ & $\begin{array}{l}228 \\
(22.2 \%)\end{array}$ & 0.772 \\
\hline CKD & $\begin{array}{l}217 \\
(23.5 \%)\end{array}$ & $\begin{array}{l}205 \\
(20.5 \%)\end{array}$ & $\begin{array}{l}221 \\
(21.8 \%)\end{array}$ & $\begin{array}{l}220 \\
(21.46 \%)\end{array}$ & 0.499 \\
\hline Metabolic syndrome & $\begin{array}{l}222 \\
(24.1 \%)\end{array}$ & $\begin{array}{l}201 \\
(20.1 \%)\end{array}$ & $\begin{array}{l}239 \\
(23.6 \%)\end{array}$ & $\begin{array}{l}216 \\
(21.1 \%)\end{array}$ & 0.095 \\
\hline Metabolic syndrome score & & & & & 0.006 \\
\hline 0 & $\begin{array}{l}127 \\
(13.8 \%)\end{array}$ & $\begin{array}{l}192 \\
(19.2 \%)\end{array}$ & $\begin{array}{l}186 \\
(18.4 \%)\end{array}$ & $\begin{array}{l}185 \\
(18.0 \%)\end{array}$ & \\
\hline
\end{tabular}




\begin{tabular}{|c|c|c|c|c|c|}
\hline & Low & Low-mid & High-mid & High & $\begin{array}{l}\mathrm{P}- \\
\text { value }\end{array}$ \\
\hline 1 & $\begin{array}{l}305 \\
(33.1 \%)\end{array}$ & $\begin{array}{l}275 \\
(27.5 \%)\end{array}$ & $\begin{array}{l}309 \\
(30.5 \%)\end{array}$ & $\begin{array}{l}314 \\
(30.6 \%)\end{array}$ & \\
\hline 2 & $\begin{array}{l}267 \\
(29.0 \%)\end{array}$ & $\begin{array}{l}331 \\
(33.1 \%)\end{array}$ & $\begin{array}{l}278 \\
(27.5 \%)\end{array}$ & $\begin{array}{l}310 \\
(30.2 \%)\end{array}$ & \\
\hline 3 & $\begin{array}{l}173 \\
(18.8 \%)\end{array}$ & $\begin{array}{l}162 \\
(16.2 \%)\end{array}$ & $\begin{array}{l}197 \\
(19.5 \%)\end{array}$ & $\begin{array}{l}179 \\
(17.5 \%)\end{array}$ & \\
\hline 4 & $\begin{array}{l}49 \\
(5.3 \%)\end{array}$ & $\begin{array}{l}39 \\
(3.9 \%)\end{array}$ & $\begin{array}{l}42 \\
(4.2 \%)\end{array}$ & 37 (3.6\%) & \\
\hline $\begin{array}{l}\text { Lack of access to necessary medical services owing to } \\
\text { economic reasons }\end{array}$ & $\begin{array}{l}100 \\
(10.9 \%)\end{array}$ & $\begin{array}{l}61 \\
(6.1 \%)\end{array}$ & $\begin{array}{l}66 \\
(6.5 \%)\end{array}$ & $30(2.9 \%)$ & $\begin{array}{l}< \\
0.001\end{array}$ \\
\hline
\end{tabular}

In the elderly age group, unlike the middle-aged group, no differences were observed across the groups in the prevalence of hypertension, diabetes, CKD, and metabolic syndrome. However, there were significant differences regarding the lack of access to necessary medical services due to economic reasons according to income level. Food safety scores in the survey showed significant differences between groups (Table 3). 
Table 3

Food security questionnaire for the Korean National Health and Nutrition Examination Survey (Investigated over 65 years).

\begin{tabular}{|c|c|c|c|c|c|}
\hline & Low & $\begin{array}{l}\text { Low- } \\
\text { mid }\end{array}$ & $\begin{array}{l}\text { High- } \\
\text { mid }\end{array}$ & High & $\begin{array}{l}\mathrm{P}- \\
\text { value }\end{array}$ \\
\hline \multicolumn{6}{|l|}{ Age $\geq 65$ years } \\
\hline 1. Food security & & & & & 0.000 \\
\hline A. We were able to eat a sufficient amount and variety of food. & $\begin{array}{l}303 \\
(32.9 \%)\end{array}$ & $\begin{array}{l}388 \\
(38.8 \%)\end{array}$ & $\begin{array}{l}468 \\
(46.2 \%)\end{array}$ & $\begin{array}{l}590 \\
(57.6 \%)\end{array}$ & \\
\hline $\begin{array}{l}\text { B. We were able to eat a sufficient amount of food, but not a } \\
\text { variety of foods. }\end{array}$ & $\begin{array}{l}480 \\
(52.1 \%)\end{array}$ & $\begin{array}{l}524 \\
(52.5 \%)\end{array}$ & $\begin{array}{l}504 \\
(49.8 \%)\end{array}$ & $\begin{array}{l}418 \\
(40.8 \%)\end{array}$ & \\
\hline $\begin{array}{l}\text { C. Because of a lack of money for food, food didn't sometimes } \\
\text { last }\end{array}$ & $\begin{array}{l}103 \\
(11.2 \%)\end{array}$ & $\begin{array}{l}68 \\
(6.8 \%)\end{array}$ & $\begin{array}{l}30 \\
(3.0 \%)\end{array}$ & $\begin{array}{l}15 \\
(1.5 \%)\end{array}$ & \\
\hline D. Because of a lack of money for food, food didn't often last & $\begin{array}{l}35 \\
(3.8 \%)\end{array}$ & $\begin{array}{l}19 \\
(1.9 \%)\end{array}$ & $\begin{array}{l}10 \\
(1.0 \%)\end{array}$ & $\begin{array}{l}2 \\
(0.2 \%)\end{array}$ & \\
\hline $\begin{array}{l}\text { 2. During the last } 12 \text { months, because of a lack of money for } \\
\text { food, how often did food not last in your household? }\end{array}$ & & & & & 0.000 \\
\hline A. Often & $\begin{array}{l}31 \\
(3.4 \%)\end{array}$ & $\begin{array}{l}17 \\
(1.7 \%)\end{array}$ & $\begin{array}{l}11 \\
(1.1 \%)\end{array}$ & $\begin{array}{l}4 \\
(0.4 \%)\end{array}$ & \\
\hline B. Sometimes & $\begin{array}{l}97 \\
(10.5 \%)\end{array}$ & $\begin{array}{l}87 \\
(8.7 \%)\end{array}$ & $\begin{array}{l}49 \\
(4.8 \%)\end{array}$ & $\begin{array}{l}23 \\
(2.2 \%)\end{array}$ & \\
\hline C. Never & $\begin{array}{l}793 \\
(86.1 \%)\end{array}$ & $\begin{array}{l}895 \\
(89.6 \%)\end{array}$ & $\begin{array}{l}952 \\
(94.1 \%)\end{array}$ & $\begin{array}{l}998 \\
(7.4 \%)\end{array}$ & \\
\hline $\begin{array}{l}\text { 3. During the last } 12 \text { months, because of a lack of money for } \\
\text { food, how often have you/others in your household worried } \\
\text { about running out food before getting money to buy more? }\end{array}$ & & & & & 0.000 \\
\hline A. Often & $\begin{array}{l}109 \\
(11.8 \%)\end{array}$ & $\begin{array}{l}62 \\
(6.2 \%)\end{array}$ & $\begin{array}{l}35 \\
(3.5 \%)\end{array}$ & $\begin{array}{l}23 \\
(2.2 \%)\end{array}$ & \\
\hline B. Sometimes & $\begin{array}{l}168 \\
(18.2 \%)\end{array}$ & $\begin{array}{l}165 \\
(16.5 \%)\end{array}$ & $\begin{array}{l}107 \\
(10.6 \%)\end{array}$ & $\begin{array}{l}61 \\
(6.0 \%)\end{array}$ & \\
\hline C. Never & $\begin{array}{l}644 \\
(69.9 \%)\end{array}$ & $\begin{array}{l}772 \\
(77.3 \%)\end{array}$ & $\begin{array}{l}870 \\
(86.0 \%)\end{array}$ & $\begin{array}{l}941 \\
(91.8 \%)\end{array}$ & \\
\hline $\begin{array}{l}\text { 4. During the last } 12 \text { months, because of a lack of money for } \\
\text { food, how often were you (you/others in your household) unable } \\
\text { to afford to eat balanced meals? }\end{array}$ & & & & & 0.000 \\
\hline A. Often & $\begin{array}{l}107 \\
(11.6 \%)\end{array}$ & $\begin{array}{l}107 \\
(10.7 \%)\end{array}$ & $\begin{array}{l}53 \\
(5.2 \%)\end{array}$ & $\begin{array}{l}29 \\
(2.8 \%)\end{array}$ & \\
\hline B. Sometimes & $\begin{array}{l}177 \\
(19.2 \%)\end{array}$ & $\begin{array}{l}153 \\
(15.3 \%)\end{array}$ & $\begin{array}{l}130 \\
(12.8 \%)\end{array}$ & $\begin{array}{l}68 \\
(6.6 \%)\end{array}$ & \\
\hline C. Never & $\begin{array}{l}637 \\
(69.2 \%)\end{array}$ & $\begin{array}{l}739 \\
(74.0 \%)\end{array}$ & $\begin{array}{l}829 \\
(81.9 \%)\end{array}$ & $\begin{array}{l}928 \\
(0.5 \%)\end{array}$ & \\
\hline
\end{tabular}

All of the food security questions were recoded into two categories [(often/sometimes vs. never) or (yes vs. no) or (almost every month/some months but not every month vs. only one or two months)]. Each item was given a score of 1 , if the answer pointed often/sometimes, yes or almost every month/some months but not every month, or 0 for the rest responses. An additive total score was created and was classified into four levels of food security: 1 ) food secure [score: 0-2 (households with children); 0-2 (households without children)]; 2) food insecure without hunger [score: 37 (households with children; 3-5 (households without children)]; 3 ) moderate food insecure with hunger (8-12; 6-8); and 4) severe food insecure with hunger (13-18; 9-10) 


\begin{tabular}{|c|c|c|c|c|c|}
\hline & Low & $\begin{array}{l}\text { Low- } \\
\text { mid }\end{array}$ & $\begin{array}{l}\text { High- } \\
\text { mid }\end{array}$ & High & $\begin{array}{l}\mathrm{P}- \\
\text { value }\end{array}$ \\
\hline $\begin{array}{l}\text { 5. During the last } 12 \text { months, because of a lack of money for } \\
\text { food, have you (you/other adults in your household) ever either } \\
\text { cut the size of your }\end{array}$ & & & & & 0.000 \\
\hline \multicolumn{6}{|l|}{ meals or skipped meals? } \\
\hline A. Yes & $\begin{array}{l}53 \\
(5.8 \%)\end{array}$ & $\begin{array}{l}22 \\
(2.2 \%)\end{array}$ & $\begin{array}{l}6 \\
(0.6 \%)\end{array}$ & $\begin{array}{l}5 \\
(0.5 \%)\end{array}$ & \\
\hline B. No & $\begin{array}{l}319 \\
(34.6 \%)\end{array}$ & $\begin{array}{l}318 \\
(31.8 \%)\end{array}$ & $\begin{array}{l}220 \\
(21.7 \%)\end{array}$ & $\begin{array}{l}122 \\
(11.9 \%)\end{array}$ & \\
\hline C. Doesn't know or refuses to answer & $\begin{array}{l}549 \\
(59.6 \%)\end{array}$ & $\begin{array}{l}659 \\
(66.0 \%)\end{array}$ & $\begin{array}{l}786 \\
(77.7 \%)\end{array}$ & $\begin{array}{l}898 \\
(87.6 \%)\end{array}$ & \\
\hline $5-1$. How often? & & & & & 0.000 \\
\hline A. Almost every month & $\begin{array}{l}18 \\
(2.0 \%)\end{array}$ & $\begin{array}{l}6 \\
(0.6 \%)\end{array}$ & $\begin{array}{l}2 \\
(0.2 \%)\end{array}$ & $\begin{array}{l}2 \\
(0.2 \%)\end{array}$ & \\
\hline B. Some months but not every month & $\begin{array}{l}16 \\
(1.7 \%)\end{array}$ & $\begin{array}{l}6 \\
(0.6 \%)\end{array}$ & $\begin{array}{l}0 \\
(0.0 \%)\end{array}$ & $\begin{array}{l}0 \\
(0.0 \%)\end{array}$ & \\
\hline C. Only one or two months & $\begin{array}{l}19 \\
(2.1 \%)\end{array}$ & $\begin{array}{l}10 \\
(1.0 \%)\end{array}$ & $\begin{array}{l}4 \\
(0.4 \%)\end{array}$ & $\begin{array}{l}3 \\
(0.3 \%)\end{array}$ & \\
\hline $\begin{array}{l}\text { 6. During the last } 12 \text { months, because of a lack of money for } \\
\text { food, have you ever eaten less than you felt you should? }\end{array}$ & & & & & 0.000 \\
\hline A. Yes & $\begin{array}{l}98 \\
(10.6 \%)\end{array}$ & $\begin{array}{l}88 \\
(8.8 \%)\end{array}$ & $\begin{array}{l}53 \\
(5.2 \%)\end{array}$ & $\begin{array}{l}24 \\
(2.3 \%)\end{array}$ & \\
\hline B. No & $\begin{array}{l}274 \\
(29.8 \%)\end{array}$ & $\begin{array}{l}252 \\
(25.2 \%)\end{array}$ & $\begin{array}{l}173 \\
(17.1 \%)\end{array}$ & $\begin{array}{l}103 \\
(10.0 \%)\end{array}$ & \\
\hline $\begin{array}{l}\text { 7. During the last } 12 \text { months, because of a lack of money for } \\
\text { food, have you ever not eaten although you were hungry? }\end{array}$ & & & & & 0.000 \\
\hline A. Yes & $\begin{array}{l}52 \\
(5.6 \%)\end{array}$ & $\begin{array}{l}24 \\
(2.4 \%)\end{array}$ & $\begin{array}{l}12 \\
(1.2 \%)\end{array}$ & $\begin{array}{l}3 \\
(0.3 \%)\end{array}$ & \\
\hline B. No & $\begin{array}{l}320 \\
(34.7 \%)\end{array}$ & $\begin{array}{l}316 \\
(31.6 \%)\end{array}$ & $\begin{array}{l}214 \\
(21.1 \%)\end{array}$ & $\begin{array}{l}124 \\
(12.1 \%)\end{array}$ & \\
\hline $\begin{array}{l}\text { 8. During the last } 12 \text { months, because of a lack of money for } \\
\text { food, have you ever lost weight because you did not eat as } \\
\text { needed? }\end{array}$ & & & & & 0.000 \\
\hline A. Yes & $\begin{array}{l}43 \\
(4.7 \%)\end{array}$ & $\begin{array}{l}27 \\
(2.7 \%)\end{array}$ & $\begin{array}{l}10 \\
(1.0 \%)\end{array}$ & $\begin{array}{l}1 \\
(0.1 \%)\end{array}$ & \\
\hline B. No & $\begin{array}{l}329 \\
(35.7 \%)\end{array}$ & $\begin{array}{l}313 \\
(31.3 \%)\end{array}$ & $\begin{array}{l}216 \\
(21.3 \%)\end{array}$ & $\begin{array}{l}126 \\
(12.3 \%)\end{array}$ & \\
\hline
\end{tabular}

All of the food security questions were recoded into two categories [(often/sometimes vs. never) or (yes vs. no) or
(almost every month/some months but not every month vs. only one or two months)]. Each item was given a score of
1 , if the answer pointed often/sometimes, yes or almost every month/some months but not every month, or 0 for the
rest responses. An additive total score was created and was classified into four levels of food security: 1 ) food secure
[score: $0-2$ (households with children); $0-2$ (households without children)]; 2 ) food insecure without hunger [score: 3-
7 (households with children; $3-5$ (households without children)]; 3 ) moderate food insecure with hunger (8-12; 6-8);
and 4) severe food insecure with hunger $(13-18 ; 9-10)$ 


\begin{tabular}{|c|c|c|c|c|c|}
\hline & Low & $\begin{array}{l}\text { Low- } \\
\text { mid }\end{array}$ & $\begin{array}{l}\text { High- } \\
\text { mid }\end{array}$ & High & $\begin{array}{l}P \text { - } \\
\text { value }\end{array}$ \\
\hline $\begin{array}{l}\text { 9. During the last } 12 \text { months, because of a lack of money for } \\
\text { food, have you (you/you or other adults in your household) ever } \\
\text { been hungry for a whole day? }\end{array}$ & & & & & 0.000 \\
\hline A. Yes & $\begin{array}{l}7 \\
(0.8 \%)\end{array}$ & $\begin{array}{l}1 \\
(0.1 \%)\end{array}$ & $\begin{array}{l}0 \\
(0.0 \%)\end{array}$ & $\begin{array}{l}0 \\
(0.0 \%)\end{array}$ & \\
\hline B. No & $\begin{array}{l}115 \\
(12.5 \%)\end{array}$ & $\begin{array}{l}99 \\
(9.9 \%)\end{array}$ & $\begin{array}{l}57 \\
(5.6 \%)\end{array}$ & $\begin{array}{l}28 \\
(2.7 \%)\end{array}$ & \\
\hline $9-1$. How often? & & & & & 0.021 \\
\hline A. Almost every month & $\begin{array}{l}1 \\
(0.1 \%)\end{array}$ & $\begin{array}{l}0 \\
(0.0 \%)\end{array}$ & $\begin{array}{l}0 \\
(0.0 \%)\end{array}$ & $\begin{array}{l}0 \\
(0.0 \%)\end{array}$ & \\
\hline B. Some months but not every month & $\begin{array}{l}2 \\
(0.2 \%)\end{array}$ & $\begin{array}{l}0 \\
(0.0 \%)\end{array}$ & $\begin{array}{l}0 \\
(0.0 \%)\end{array}$ & $\begin{array}{l}0 \\
(0.0 \%)\end{array}$ & \\
\hline C. Only one or two months & $\begin{array}{l}4 \\
(0.4 \%)\end{array}$ & $\begin{array}{l}1 \\
(0.1 \%)\end{array}$ & $\begin{array}{l}0 \\
(0.0 \%)\end{array}$ & $\begin{array}{l}0 \\
(0.0 \%)\end{array}$ & \\
\hline $\begin{array}{l}\text { 10. During the last } 12 \text { months, because of a lack of or being } \\
\text { frugal about money for food, how often have you fed your } \\
\text { child/children with only one or two kinds of low-cost food? }\end{array}$ & & & & & 0.000 \\
\hline A. Often & $\begin{array}{l}9 \\
(1.0 \%)\end{array}$ & $\begin{array}{l}5 \\
(0.5 \%)\end{array}$ & $\begin{array}{l}7 \\
(0.7 \%)\end{array}$ & $\begin{array}{l}3 \\
(0.3 \%)\end{array}$ & \\
\hline B. Sometimes & $\begin{array}{l}10 \\
(1.1 \%)\end{array}$ & $\begin{array}{l}14 \\
(1.4 \%)\end{array}$ & $\begin{array}{l}13 \\
(1.3 \%)\end{array}$ & $\begin{array}{l}7 \\
(0.7 \%)\end{array}$ & \\
\hline C. Never & $\begin{array}{l}57 \\
(6.2 \%)\end{array}$ & $\begin{array}{l}58 \\
(5.8 \%)\end{array}$ & $\begin{array}{l}92 \\
(9.1 \%)\end{array}$ & $\begin{array}{l}179 \\
(17.5 \%)\end{array}$ & \\
\hline $\begin{array}{l}\text { 11. During the last } 12 \text { months, because of a lack of money, how } \\
\text { often did you not feed your child/children a balanced meal? }\end{array}$ & & & & & 0.000 \\
\hline A. Often & $\begin{array}{l}10 \\
(1.1 \%)\end{array}$ & $\begin{array}{l}3 \\
(0.3 \%)\end{array}$ & $\begin{array}{l}9 \\
(0.9 \%)\end{array}$ & $\begin{array}{l}3 \\
(0.3 \%)\end{array}$ & \\
\hline B. Sometimes & $\begin{array}{l}11 \\
(1.2 \%)\end{array}$ & $\begin{array}{l}14 \\
(1.4 \%)\end{array}$ & $\begin{array}{l}8 \\
(0.8 \%)\end{array}$ & $\begin{array}{l}14 \\
(1.4 \%)\end{array}$ & \\
\hline C. Never & $\begin{array}{l}55 \\
(6.0 \%)\end{array}$ & $\begin{array}{l}60 \\
(6.0 \%)\end{array}$ & $\begin{array}{l}95 \\
(9.4 \%)\end{array}$ & $\begin{array}{l}172 \\
(16.8 \%)\end{array}$ & \\
\hline $\begin{array}{l}\text { 12. During the last } 12 \text { months, because of a lack of money, how } \\
\text { often did you not feed your child/children as needed? }\end{array}$ & & & & & 0.000 \\
\hline A. Often & $\begin{array}{l}7 \\
(0.8 \%)\end{array}$ & $\begin{array}{l}5 \\
(0.5 \%)\end{array}$ & $\begin{array}{l}5 \\
(0.5 \%)\end{array}$ & $\begin{array}{l}1 \\
(0.1 \%)\end{array}$ & \\
\hline B. Sometimes & $\begin{array}{l}12 \\
(1.3 \%)\end{array}$ & $\begin{array}{l}7 \\
(0.7 \%)\end{array}$ & $\begin{array}{l}11 \\
(1.1 \%)\end{array}$ & $\begin{array}{l}8 \\
(0.8 \%)\end{array}$ & \\
\hline
\end{tabular}

All of the food security questions were recoded into two categories [(often/sometimes vs. never) or (yes vs. no) or (almost every month/some months but not every month vs. only one or two months)]. Each item was given a score of 1 , if the answer pointed often/sometimes, yes or almost every month/some months but not every month, or 0 for the rest responses. An additive total score was created and was classified into four levels of food security: 1 ) food secure [score: 0-2 (households with children); 0-2 (households without children)]; 2) food insecure without hunger [score: 37 (households with children; $3-5$ (households without children)]; 3 ) moderate food insecure with hunger (8-12; 6-8); and 4) severe food insecure with hunger (13-18;9-10) 


\begin{tabular}{|c|c|c|c|c|c|}
\hline & Low & $\begin{array}{l}\text { Low- } \\
\text { mid }\end{array}$ & $\begin{array}{l}\text { High- } \\
\text { mid }\end{array}$ & High & $\begin{array}{l}\mathrm{P}- \\
\text { value }\end{array}$ \\
\hline C. Never & $\begin{array}{l}57 \\
(6.2 \%)\end{array}$ & $\begin{array}{l}65 \\
(6.5 \%)\end{array}$ & $\begin{array}{l}96 \\
(9.5 \%)\end{array}$ & $\begin{array}{l}180 \\
(17.6 \%)\end{array}$ & \\
\hline $\begin{array}{l}\text { 13. During the last } 12 \text { months, because of a lack of money, have } \\
\text { you ever cut the size of your (child's/children's) meals? }\end{array}$ & & & & & 0.612 \\
\hline A. Yes & $\begin{array}{l}3 \\
(0.3 \%)\end{array}$ & $\begin{array}{l}0 \\
(0.0 \%)\end{array}$ & $\begin{array}{l}2 \\
(0.2 \%)\end{array}$ & $\begin{array}{l}2 \\
(0.2 \%)\end{array}$ & \\
\hline B. No & $\begin{array}{l}22 \\
(2.4 \%)\end{array}$ & $\begin{array}{l}25 \\
(2.5 \%)\end{array}$ & $\begin{array}{l}22 \\
(2.2 \%)\end{array}$ & $\begin{array}{l}18 \\
(1.8 \%)\end{array}$ & \\
\hline $\begin{array}{l}\text { 14. During the last } 12 \text { months, because of a lack of money, have } \\
\text { your child/children ever skipped meals? }\end{array}$ & & & & & 0.461 \\
\hline A. Yes & $\begin{array}{l}0 \\
(0.0 \%)\end{array}$ & $\begin{array}{l}0 \\
(0.0 \%)\end{array}$ & $\begin{array}{l}1 \\
(0.1 \%)\end{array}$ & $\begin{array}{l}2 \\
(0.2 \%)\end{array}$ & \\
\hline B. No & $\begin{array}{l}25 \\
(2.7 \%)\end{array}$ & $\begin{array}{l}25 \\
(2.5 \%)\end{array}$ & $\begin{array}{l}23 \\
(2.3 \%)\end{array}$ & $\begin{array}{l}18 \\
(1.8 \%)\end{array}$ & \\
\hline \multicolumn{6}{|l|}{$14-1$. How often? } \\
\hline \multicolumn{6}{|l|}{ A. Almost every month } \\
\hline \multicolumn{6}{|l|}{ B. Some months but not every month } \\
\hline C. Only one or two months & & & & & \\
\hline
\end{tabular}

15. During the last 12 months, because of a lack of money, have your child/children ever not eaten although they were hungry?

$\begin{array}{lllll}\text { A. Yes } & 2 & 2 & 2 & 2 \\ & (0.2 \%) & (0.2 \%) & (0.2 \%) & (0.2 \%) \\ \text { B. No } & 23 & 23 & 22 & 18 \\ & (2.5 \%) & (2.3 \%) & (2.2 \%) & (1.8 \%)\end{array}$

16. During the last 12 months, because of a lack of money, have your child/children ever been hungry for a whole day?
A. Yes
B. No

All of the food security questions were recoded into two categories [(often/sometimes vs. never) or (yes vs. no) or (almost every month/some months but not every month vs. only one or two months)]. Each item was given a score of 1 , if the answer pointed often/sometimes, yes or almost every month/some months but not every month, or 0 for the rest responses. An additive total score was created and was classified into four levels of food security: 1) food secure [score: 0-2 (households with children); 0-2 (households without children)]; 2) food insecure without hunger [score: 37 (households with children; $3-5$ (households without children)]; 3) moderate food insecure with hunger (8-12;6-8); and 4 ) severe food insecure with hunger $(13-18 ; 9-10)$ 


\begin{tabular}{|c|c|c|c|c|c|}
\hline & Low & $\begin{array}{l}\text { Low- } \\
\text { mid }\end{array}$ & $\begin{array}{l}\text { High- } \\
\text { mid }\end{array}$ & High & $\begin{array}{l}\mathrm{P} \text { - } \\
\text { value }\end{array}$ \\
\hline Total score of food security & $\begin{array}{l}1.1 \pm \\
1.9\end{array}$ & $\begin{array}{l}0.8 \pm \\
1.5\end{array}$ & $\begin{array}{l}0.5 \pm \\
1.2\end{array}$ & $\begin{array}{l}0.3 \pm \\
0.9\end{array}$ & 0.000 \\
\hline Four levels of food security & & & & & 0.000 \\
\hline I. Food secure & $\begin{array}{l}762 \\
(82.7 \%)\end{array}$ & $\begin{array}{l}875 \\
(87.6 \%)\end{array}$ & $\begin{array}{l}935 \\
(92.4 \%)\end{array}$ & $\begin{array}{l}982 \\
(95.8 \%)\end{array}$ & \\
\hline II. Food insecure without hunger & $\begin{array}{l}119 \\
(12.9 \%)\end{array}$ & $\begin{array}{l}111 \\
(11.1 \%)\end{array}$ & $\begin{array}{l}72 \\
(7.1 \%)\end{array}$ & $\begin{array}{l}42 \\
(4.1 \%)\end{array}$ & \\
\hline III. Moderate food insecure with hunger & $\begin{array}{l}37 \\
(4.0 \%)\end{array}$ & $\begin{array}{l}13 \\
(1.3 \%)\end{array}$ & $\begin{array}{l}5 \\
(0.5 \%)\end{array}$ & $\begin{array}{l}1 \\
(0.1 \%)\end{array}$ & \\
\hline IV. Severe food insecure with hunger & $\begin{array}{l}3 \\
(0.3 \%)\end{array}$ & $\begin{array}{l}0 \\
(0.0 \%)\end{array}$ & $\begin{array}{l}0 \\
(0.0 \%)\end{array}$ & $\begin{array}{l}0 \\
(0.0 \%)\end{array}$ & \\
\hline \multicolumn{6}{|c|}{$\begin{array}{l}\text { All of the food security questions were recoded into two categories [(often/sometimes vs. never) or (yes vs. no) or } \\
\text { (almost every month/some months but not every month vs. only one or two months)]. Each item was given a score of } \\
1 \text {, if the answer pointed often/sometimes, yes or almost every month/some months but not every month, or } 0 \text { for the } \\
\text { rest responses. An additive total score was created and was classified into four levels of food security: } 1 \text { ) food secure } \\
\text { [score: } 0-2 \text { (households with children); } 0-2 \text { (housseholds without children)]; } 2 \text { ) food insecure without hunger [score: } 3- \\
7 \text { (households with children; } 3-5 \text { (households without children)]; } 3 \text { ) moderate food insecure with hunger }(8-12 ; 6-8) \text {; } \\
\text { and 4) severe food insecure with hunger }(13-18 ; 9-10)\end{array}$} \\
\hline
\end{tabular}


Table 4

A. Effect of income level on the prevalence of hypertension.

\begin{tabular}{|c|c|c|c|c|c|c|c|c|}
\hline \multirow[b]{2}{*}{ Under 65 years of age } & \multicolumn{2}{|c|}{ Model 1} & \multicolumn{2}{|c|}{ Model 2} & \multicolumn{2}{|c|}{ Model 3} & \multicolumn{2}{|c|}{ Model 4} \\
\hline & $\begin{array}{l}\text { Odds } \\
\text { ratio }\end{array}$ & $95 \% \mathrm{Cl}$ & $\begin{array}{l}\text { Odds } \\
\text { ratio }\end{array}$ & $95 \% \mathrm{Cl}$ & $\begin{array}{l}\text { Odds } \\
\text { ratio }\end{array}$ & $95 \% \mathrm{Cl}$ & $\begin{array}{l}\text { Odds } \\
\text { ratio }\end{array}$ & $95 \% \mathrm{Cl}$ \\
\hline \multicolumn{9}{|l|}{$\begin{array}{l}\text { Income (ref. high income } \\
\text { level) }\end{array}$} \\
\hline Low & 1.49 & $\begin{array}{l}1.19 \sim \\
1.86\end{array}$ & 1.55 & $\begin{array}{l}1.23 \sim \\
1.94\end{array}$ & 1.15 & $\begin{array}{l}0.91 \sim \\
1.46\end{array}$ & 1.12 & $\begin{array}{l}0.82 \sim \\
1.52\end{array}$ \\
\hline Low-mid & 1.25 & $\begin{array}{l}1.00 \sim \\
1.56\end{array}$ & 1.26 & $\begin{array}{l}1.01 \sim \\
1.58\end{array}$ & 1.01 & $\begin{array}{l}0.80 \sim \\
1.27\end{array}$ & 0.93 & $\begin{array}{l}0.69 \sim \\
1.24\end{array}$ \\
\hline High-mid & 1.26 & $\begin{array}{l}1.01 \sim \\
1.58\end{array}$ & 1.27 & $\begin{array}{l}1.02 \sim \\
1.59\end{array}$ & 1.12 & $\begin{array}{l}0.89 \sim \\
1.41\end{array}$ & 1.05 & $\begin{array}{l}0.79 \sim \\
1.41\end{array}$ \\
\hline $\begin{array}{l}\text { Food security (ref. Food } \\
\text { secure) }\end{array}$ & & & 0.78 & $\begin{array}{l}0.56 \sim \\
1.07\end{array}$ & 0.72 & $\begin{array}{l}0.51 \sim \\
0.99\end{array}$ & 0.76 & $\begin{array}{l}0.50 \sim \\
1.15\end{array}$ \\
\hline Over 65 years of age & $\begin{array}{l}\text { Odds } \\
\text { ratio }\end{array}$ & $95 \% \mathrm{Cl}$ & $\begin{array}{l}\text { Odds } \\
\text { ratio }\end{array}$ & $95 \% \mathrm{Cl}$ & $\begin{array}{l}\text { Odds } \\
\text { ratio }\end{array}$ & $95 \% \mathrm{Cl}$ & $\begin{array}{l}\text { Odds } \\
\text { ratio }\end{array}$ & $95 \% \mathrm{Cl}$ \\
\hline \multicolumn{9}{|l|}{$\begin{array}{l}\text { Income (ref. high income } \\
\text { level) }\end{array}$} \\
\hline Low & 0.98 & $\begin{array}{l}0.82 \sim \\
1.18\end{array}$ & 0.98 & $\begin{array}{l}0.81 \sim \\
1.17\end{array}$ & 0.88 & $\begin{array}{l}0.73 \sim \\
1.07\end{array}$ & 0.85 & $\begin{array}{l}0.68 \sim \\
1.06\end{array}$ \\
\hline Low-mid & 1.10 & $\begin{array}{l}0.92 \sim \\
1.32\end{array}$ & 1.10 & $\begin{array}{l}0.91 \sim \\
1.31\end{array}$ & 1.00 & $\begin{array}{l}0.83 \sim \\
1.21\end{array}$ & 1.05 & $\begin{array}{l}0.84 \sim \\
1.30\end{array}$ \\
\hline High - mid & 1.01 & $\begin{array}{l}0.84 \sim \\
1.20\end{array}$ & 1.01 & $\begin{array}{l}0.84 \sim \\
1.20\end{array}$ & 0.95 & $\begin{array}{l}0.79 \sim \\
1.14\end{array}$ & 0.98 & $\begin{array}{l}0.79 \sim \\
1.03\end{array}$ \\
\hline $\begin{array}{l}\text { Food security (ref. Food } \\
\text { secure) }\end{array}$ & & & 1.05 & $\begin{array}{l}0.84 \sim \\
1.30\end{array}$ & 1.00 & $\begin{array}{l}0.81 \sim \\
0.93\end{array}$ & 0.90 & $\begin{array}{l}0.70 \sim \\
1.16\end{array}$ \\
\hline
\end{tabular}

\section{Regression analysis based on income level.}

In the middle age group, the relative risk of hypertension according to income level was 1.49 times $(95 \% \mathrm{Cl} 1.19-1.86)$ and 1.55 times $(95 \% \mathrm{Cl} 1.23-1.94)$ higher in the low-income group, compared with that in the high-income group in Models 1 and 2, respectively (Table 4A). However, since there was no significant increase in risk in Models 3 and 4, the effect of education level and food safety on the prevalence of hypertension could not be confirmed. The relative risk of diabetes according to income level was 1.63 times (95\% $\mathrm{Cl} 1.17-2.28)$ and 1.61 times $(95 \% \mathrm{Cl} 1.15-2.27)$ higher in the low-income group, compared with that in the high-income group in Model 1 and 2, respectively; however, the effect of education level and food safety could not be confirmed (Table 4B). There was no increase in the relative risk of CKD in any of the Models.

In the elderly age group, no increase in the relative risk of hypertension or diabetes in Model 1-4 was observed. The effect of income level, education level, and food security on the prevalence of hypertension and diabetes could not be identified. However, the relative risk of CKD due to food insecurity increased by 1.33 times $(95 \% \mathrm{Cl} 1.00-1.74)$ and 1.34 times (95\% Cl 1.01-1.75) in Models 2 and 3, respectively. (Table 4C).

\section{Discussion}


The relationship between socioeconomic status and chronic diseases has been of great interest in western society for several years. Socioeconomic factors comprise factors such as education and income level, occupation, and health behavior. Health behaviors are related to physical activity, avoidance of drinking and smoking, maintaining food safety, and obesity. Our study showed that among other socioeconomic factors, income level affects the prevalence of hypertension and diabetes in the $<65$ years population. These relationships were not altered after being adjusted for food safety. However, we found that the effect of income level on the prevalence of chronic disease was diminished after adjusting for factors related to education level and health behaviors such as blood pressure and abdominal

circumference. This finding could reflect that health behaviors such as smoking, abdominal circumference, and BMI differ by income level. Moreover, this finding is similar to those of previous studies, which present differences in the prevalence of chronic diseases by socioeconomic status in western societies $[6,7,8]$. It is also consistent with studies that emphasize the importance of health behavior [9]. However, this finding contrasts those of other studies, which reveal that health behaviors do not affect the relationship between social class and the prevalence of chronic diseases in the Korean population [10].

In the elderly age group over 65 years, although the level of education varied by income, the effect on the prevalence of diabetes and hypertension could not be confirmed. Moreover, unlike that of the population under 65 years, no increase in health-risk behaviors was observed, which resulted in an increase in the risk of diabetes and hypertension such as BMI and waist circumference in the low-income group. Although food insecurity and smoking rates tended to be higher among those with lower income, other health behaviors did not increase in the elderly group. This may explain why income level does not affect the prevalence of diabetes and hypertension in the elderly.

It is well known that socioeconomic level influences kidney health, as the prevalence of chronic metabolic diseases causing kidney disease, as well as drinking and physical activity is closely related to socioeconomic status [11, 12, 13]. Furthermore, food insecurity may be a risk factor for independent kidney disease progression as well as the occurrence of chronic diseases $[14,15]$. Insufficient intake of fruits and vegetables and consumption of high-energy processed foods leads to an increase in salt and saturated fatty acid intake, which is believed to aggravate kidney disease by increasing the dietary acid load $[16,17]$. Contrary to the results of previous studies, our study could not identify the effect of income and education levels on CKD. However, we found that food insecurity increases the risk of CKD in the elderly population (over age 65 years); moreover, the effect of food instability was attenuated when adjusted for height, abdominal circumference, and calorie intake.

Our study shows that the difference in chronic diseases by income level is similar to that found in western societies in the $>65$ years Korean population. Therefore, we could infer that with increasing health risk behavior such as obesity and smoking in the lower-income level group, there is a greater need for social efforts to improve these behaviors. However, there was no difference in factors related to health-risk behaviors by income level in the elderly population. These characteristics would allow for socioeconomic factors like food instability to have a greater effect than preexisting traditional metabolic causes. This is supported by the fact that food insecurity could worsen glycemic control in diabetic patients [18]. Some studies have also shown a relationship between poverty and food insecurity [19, 20], while others have revealed that poorer areas have a higher incidence of ESRD [21, 22].

Our study has limitations in that occupational factors are not included among the socioeconomic status factors; moreover, cardiovascular diseases are not included among chronic diseases.

In conclusion, we found that food insecurity is associated with CKD in the Korean elderly population. Considering this finding in combination with disparities in access to essential medical services according to income level, we need to establish a different approach to tackle food security in the elderly population, compared with that of the middle age group.

Page $13 / 16$ 


\section{Methods}

\section{Study population and baseline data}

To study the association between household income, food security, and the prevalence of chronic disease, we used data based on the KNHANES, which has been conducted by the Division of Health and Nutritional Survey in the Korean Centers for Disease Control and Prevention from 2012-2015. The KNHANES is a population-based, cross-sectional survey with nationally representative samples of the civilian non-institutionalized Korean population. Among the 31,017 individuals surveyed from 2012-2015, 15,945 people were included, after eliminating those who did not complete the survey. We measured the propensity score matching by income level, which was categorized into four levels, and sex. Using the propensity score, participants were divided by age ( $<65$ years old: middle-aged adult, $>65$ years old: elderly age adult) and gender. Finally, 3,957 participants were qualified for statistical analysis (Table 1).

\section{Socioeconomic and clinical measurements}

Medical history and demographic data were collected through three component surveys: a health interview, health examination, and nutrition survey. Annual household income was divided into four quartiles using health interview data. The educational status was stratified into three categories including elementary, middle, high school, and college, based on the academic background. Body mass index (BMI) was calculated from anthropometric data, where weight was divided by height squared. Diabetes was defined by self-report or measured hemoglobin A1c level 6.5\%. Hypertension was defined as self-report, measured average systolic blood pressure $>140 \mathrm{~mm} \mathrm{Hg}$, measured average diastolic blood pressure $>90 \mathrm{~mm} \mathrm{Hg}$, or reported using antihypertensive medications. CKD is defined as estimated glomerular filtration rate (eGFR) of 15 to $59 \mathrm{~mL} / \mathrm{min} / 1.73 \mathrm{~m}^{2}$ or urinary albumin creatinine ratio (ACR) $>30 \mathrm{mg} / \mathrm{g}$.

\section{Food security}

To assess food security, we used the Food security questionnaire for Korean National Health and the Nutrition Examination Survey that addresses dietary behaviors, food frequency. and food intake. The dietary behavior questionnaire includes meal skipping, eating out, eating with family, taking dietary supplements, nutrition education, use of food labeling, and food security. The food frequency questionnaire comprises 63 food items that are key sources of energy and nutrients (Table 3).

\section{Ethical considerations}

This study was based on data collected during the KNHANES and the secondary analysis of a large open data set. The KNHANES methodology has been presented in detail previously and further details "The 5th KNHANES Sample Design" and accessible at https://knhanes.cdc.go.kr/knhanes/index.do

\section{Statistical analysis}

Regression analysis was performed with four models to identify the effects of income level, food security, and education level on chronic diseases as socioeconomic factors. In each age group, participants were matched by quartile of household income (low, low-mid, high-mid, high) and sex on base of propensity score. Baseline characteristics according to income level in each age group were compared by the Mann-Whitney $U$ test for continuous variables and the $\nabla 2$ test for categorical variables. Multivariate logistic regression analysis was performed with four models to identify the effects of income level, food security, and education level on chronic diseases as socioeconomic factors yielding odds ratio (OR).

\section{Declarations}


Acknowledgments

None

Author contribution statement

Research idea and study design: KDY, HSK; data acquisition: HH, JHS, HSK, KDY; data analysis/interpretation: HH, JHS, HSK, KDY; supervision or mentorship: KDY. Each author contributed important intellectual content during manuscript drafting or revision. All authors read and approved the final manuscript.

\section{Competing Interests}

The authors declare no competing interests.

\section{Data Availability}

The datasets generated during and/or analysed during the current study are available from the corresponding author on reasonable request.

\section{References}

1. Chetty R, Stepner M, Abraham S, et al. The association between income and life expectancy in the United States, 2001-2014. JAMA. 2016.315:1750-66.

2. Shoham DA, Vupputuri S, Kaufman JS, et al. Kidney disease and the cumulative burden of life course socioeconomic conditions: the Atherosclerosis Risk in Communities (ARIC) study. Soc Sci Med. 2008,67:1311-20.

3. Crews DC, Kuczmarski MF, Grubbs V, et al. Effect of food insecurity on chronic kidney disease in lower-income Americans. American journal of nephrology. 2014;39(1):27-35

4. Banerjee, T.; Crews, D.C.; Wesson, D.E, et al. CDC CKD Surveillance Team. Food Insecurity, CKD, and Subsequent ESRD in US Adults. Am. J. Kidney Dis. 2017

5. Amano, H., Fukuda, Y., Kitashima1, C., Yokoo, T. and Yamaoka, K et al. Individual Income Status Correlates with Chronic Kidney Disease in Japan beyond Metabolic Risk Factors: Cross Sectional Study. Health, 9, 1516-1528. 2007

6. AEJM Cavelaars, AE Kunst, JJM Geurt et al. Morbidity differences by occupational class among men in seven European countries: an application of the Erikson-Goldthorpe social class scheme. International Journal of Epidemiology 1998,27:222-230

7. TAPANI VALKONEN, ARI-PEKKA SIHVONEN, EERO LAHELMA et al. HEALTH EXPECTANCY BY LEVEL OF EDUCATION IN FINLAND. Soc. Sci. Med. Vol. 44, No. 6, pp. 801-808, 1997

8. K STRONKS, H VAN DE MHEEN, J VAN DEN BOS AND J P MACKENBACH et al. The Interrelationship between Income, Health and Employment Status. International Journal of Epidemiology, 1997

9. Nancy E. Adler, W. Thomas Boyce, Margaret A et al. Socioeconomic Inequalities in Health: No Easy Solution. JAMA. 199,269:3140-3145

10. Mia Son. The Relationship of Social Class and Health Behaviors with Morbidity in Korea. Korean J prev med, 2002

11. Fukuda, Y., Nakamura, K. and Takano, T et al. Accumulation of Health Risk Behaviours Is Associated with Lower Socioeconomic Status and Women's Urban Residence: A Multilevel Analysis in Japan. BMC Public Health, 2005

12. Gray, L. and Leyland, A.H, et al. A Multilevel Analysis of Diet and Socio-Economic Status in Scotland: Investigating the 'Glasgow Effect'. Public Health Nutrition, 12, 1351-1358, 2009

13. Vart, P., Gansevoort, R.T., Crews, D.C., Reijneveld, S.A. and Bültmann, U, et al. Mediators of the Association between Low Socioeconomic Status and Chronic Kidney Disease in the United States. American Journal of Epidemiology, 181, 
385-396, 2015

14. Laraia BA. Food insecurity and chronic disease. Adv Nutr. 2013

15. Seligman HK, Laraia BA, Kushel M. Food insecurity is associated with chronic disease among low-income NHANES participants. J Nutr. 2010

16. Banerjee T, Crews D, Wesson D, et al. High dietary acid load predicts ESRD among adults with CKD. J Am Soc Nephrol. 2015

17. Scialla JJ, Anderson CAM. Dietary acid load: a novel nutritional target in chronic kidney disease? Adv Chronic Kidney Dis. 2013

18. Seligman HK, Bindman AB, Vittinghoff E, Kanaya AM, Kushel MB. Food insecurity is associated with diabetes mellitus: results from the National Health Examination and Nutrition Examination Survey (NHANES) 1999-2002. J Gen Intern Med. 2007,22:1018-1023.

19. Franco M, Diez Roux AV, Glass TA, Caballero B, Brancati FL. Neighborhood characteristics and availability of healthy foods in Baltimore. Am J Prev Med. 2008;35(6): 561-567.

20. Horowitz CR, Colson KA, Hebert PL, Lancaster K. Barriers to buying healthy foods for people with diabetes: evidence of environmental disparities. Am J Public Health. 2004;94(9): 1549-1554.

21. Garrity BH, Kramer H, Vellanki K, Leehey D, Brown J, Shoham DA. Time trends in the association of ESRD incidence with area-level poverty in the US population. Hemodial Int. 2016, 20:78-83.

22. Lipworth L, Mumma MT, Cavanaugh KL, et al. Incidence and predictors of end stage renal disease among low-income blacks and whites. PloS One. 2012;7(10):e48407. 